\title{
Editorials
}

Orlando Hung MD FRCPC, Joanna Mills PhD

\section{Predictions and clinical decisions: a fine balance}

M ORE than 3000 years ago, during the Shang Dynasty, Chinese sages predicted the future by casting oracle bones. Noah had it easier. He got his prediction straight from the ALMIGHTY and so he knew exactly what to expect. Our desire to know the future has not significantly changed and forecasting outcomes remains paramount to our daily living. Every day, financial experts predict and speculate upon the rise and fall of stocks. Based on sophisticated satellite mappings, meteorologists around the world predict the weather. While critical decisions are often made based on these predictions, serious and sometimes fatal errors remain part of the process. For example, earlier this year, the supercomputer in United States Weather Service made a "trivial" error and failed to forecast snow - at a time when, in fact, a blizzard roared through the east coast. The blizzard paralyzed many Eastern States and killed at least six people. Errors, and sometimes fatal errors, are inherent to any predictions.

The prediction of a complex dynamic variable, such as the upper airway of a patient for laryngoscopic intubation, is not an easy task. The difficulty lies in the search for a fine balance between simplicity and reliability of the airway assessment. In clinical settings, the assessment should be simple and convenient to the clinician, and yet, it should be sufficiently robust with a high predictive power. A simple airway evaluation, such as Mallampatti classification, ${ }^{1}$ has been widely employed to predict a difficult laryngoscopic intubation. However, recent reports suggest that it is unreliable with a low sensitivity and specificity. ${ }^{2-3}$ An objective airway assessment utilizing radiological evaluation has been suggested to be more reliable and reproducible. ${ }^{4}$ However, its implementation at the bedside is impractical.

Clearly, there is no single airway parameter which can reliably predict a difficult airway or laryngoscopic intubation. Evidence from peer-reviewed literature suggests that multi-variable bedside airway assessment may provide a more reliable method of predicting difficult laryngoscopic intubation. ${ }^{5-6}$ In this issue, Karkouti et al. . report a multi-variable assessment of the upper airway to predict difficult laryngoscopic intubation using logistic regression. The multi-variable analysis identifies three simple bedside tests (mouth opening, chin protrusion, and atlanto-occipital extension) as most useful airway parameters to predict difficult tracheal intubation. The investigators compare their predictive methods in terms of three parameters: positive predictive value, sensitivity, and specificity. When a predicted probability of $>0.2$ is used to indicate difficult intubation, the multi-variable model has a $86.8 \%$ sensitivity and $96.0 \%$ specificity. While this prediction model is highly accurate, the investigators state that a considerable number of patients may be misclassified. The investigators stress the importance of cost-effectiveness consideration entailed in a prediction model. A false negative result may expose patients to increased perioperative risk (a decrease in effectiveness), whereas a false positive result may subject the patient to unnecessary alternative intubation technique (a potential increase in cost).

Multi-variable methods of statistical analysis are widely used tools in statistics and can be useful in understanding complex relationships. Logistic regression (as chosen by Karkouti et al.) is one of many multi-variable methods available for dichotomous outcomes. Others include discriminant and classification analysis. ${ }^{8}$ The choice of an appropriate method is highly application dependent. It is important that the assumptions and limitations of the selected method are understood. For logistic regression, one must interpret the estimated coefficients carefully, be aware of the possibility of collinearity, and appropriately investigate goodness-of-fit. Having acknowledged such limitations, these methods can provide reliable prediction. Studies have shown that the efficiency and

\footnotetext{
From the Department of Anesthesia, Mathematics and Biostatistics, Dalhousie University, Halifax, Nova Scotia, Canada. Address correspondence to: Dr. Orlando Hung, Department of Anaesthesia, QEII Health Sciences Centre, Victoria General Site, 1278 Tower Road, Halifax, Nova Scotia, B3H 2 Y9 E-mail: hungorla@is.dal.ca
} 
accuracy of prediction can be improved by using straight criteria or rule. ${ }^{9}$ A clear definition of the outcome (e.g. a difficult laryngoscopic intubation), a precise predictive finding (e.g. the Mallampatti score), and the blinded assessment of outcome (free of bias) have been suggested. While these rules may help to improve the accuracy, the risk of error may still remain. Depending on the threshold chosen, Wilson et al. ${ }^{10}$ had shown that the rules might allow the detection of more patients with "difficult" laryngoscopies (high sensitivity) at a cost of falsely identifying more patients who do not have "difficult" laryngoscopies (low specificity). Similarly, Jacobson et al. ${ }^{11}$ showed that by increasing the specificity of the tests, the number of false positive classifications could be reduced. Unfortunately, this would have led to missing of patients with a difficult laryngoscopy and intubation. Ideally, we would like both high sensitivity and specificity with a predictive method. Unfortunately, we must reach a compromise and consequently should concern ourselves with the type of error that we feel is more severe. In other words, is it more acceptable to identify correctly patients who have difficult laryngoscopies even if it means falsely identifying a few patients who do not have "difficult" laryngoscopies, rather than miss a few patients who had "difficult" laryngoscopies? While most would agree that the price of missing a "few" difficult laryngoscopic intubations is far higher than falsely identifying patients with difficult airway, one should minimize the considerable stress that a patient has to go through for an alternative intubating technique, such as an awake fibreoptic intubation. Obviously, the decision is an individual one and the clinician must decide what is most appropriate to the patient without sacrificing safety.

It should be emphasized that while there is no perfect prediction for any outcome, it serves to help us to plan strategies to manage difficult situations. Reliability depends on the adherence to strict criteria and a sense of realism. At best, there is marked individual variability in measurement of the airway parameters. Prediction tools are helpful but only when used in conjunction with good clinical judgement. We all must acknowledge the inevitability of prediction error and be prepared for the worse case scenario, such as being unable to provide adequate ventilation and oxygenation to a patient. We must equip ourselves with a variety of alternative airway techniques and always be ready for the inevitable error. Noah had it easy. He was told what to do and how to do it. The rest of us are not so fortunate.

\section{Prédictions et décisions cliniques : un juste équilibre}

Il y a plus de 3000 ans, sous la dynastie des Shang, les sages chinois prédisaient l'avenir en lançant des ossements divinatoires. Ce fut plus facile pour Noé. La prédiction est venue directement du Tout-Puissant, de sorte qu'il savait exactement à quoi s'attendre. Notre désir de connaître l'avenir n'a pas vraiment changé et la prévision des résultats demeure primordiale dans notre vie quotidiennne. Chaque jour, les experts financiers font des prédictions et spéculent sur la hausse et la baisse du marché boursier. Les météorologistes du monde entier s'appuient sur des cartes sophistiquées, établies par satellite, pour prédire la température. Des décisions cruciales sont souvent prises sur la base de ces prédictions, mais des erreurs graves et quelquefois fatales demeurent possibles. Ainsi, plus tôt cette année, le superordinateur du United States Weather Service a fait une erreur «banale» et n'a pu prévoir de la neige au moment où, de fait, une tempête faisait rage sur la côte est. En plus de paralyser de nombreux États de l'est, cette tempête a tué au moins six personnes. Les erreurs, quelquefois fatales, sont inhérentes à toute prédiction.

La prévision d'une variable dynamique complexe, comme les voies aériennes supérieures d'un patient qui doit subir une intubation laryngoscopique, n'est pas une tâche facile. La difficulté réside dans la recherche d'un juste équilibre entre la simplicité et la fiabilité d'une évaluation de l'intubation. En clinique, l'évaluation doit être simple et pratique pour le médecin et, malgré tout, suffisamment fiable et comporter une grande valeur prédictive. Une évaluation simple de l'intubation, comme la classification de Mallampatti, ${ }^{1}$ a été largement utilisée pour prévoir des difficultés $\mathrm{d}$ 'intubation laryngoscopique. Cependant, des articles récents laissent croire que cette méthode est peu fiable, ayant une faible sensibilité et spécificité. ${ }^{2-3}$ Un examen objectif à l'aide d'une évaluation radiologique a été recommandé comme mesure plus sûre et reproductible. ${ }^{4}$ Toutefois, sa mise en oeuvre clinique est peu réaliste.

En fait, il n'existe pas de paramètre unique qui permette de prédire fidèlement une difficulté d'intubation laryngoscopique. La littérature scientifique révisée suggère qu'un examen clinique multifactoriel peut per- 
mettre de mieux prédire des difficultés d'intubation laryngoscopique. ${ }^{5-6}$ Dans le présent numéro, Karkouti et coll. ${ }^{7}$ font état d'un examen multivarié des voies aériennes supérieures à l'aide de régression logistique. L'analyse multivariée reconnaît trois tests cliniques simples (l'ouverture de la bouche, la protrusion du menton et l'extension atlanto-occipitale) comme paramètres d'intubation les plus utiles pour prédire des difficultés. Les chercheurs comparent leurs méthodes prédictives selon trois paramètres : la valeur prédictive, la sensibilité et la spécificité. Lorsqu'une probabilité prévue de $\geq 0,2$ est utilisée pour indiquer une difficulté d'intubation, le modèle multivarié présente une sensibilité de 86,8 \% et une spécificité de 96,0\%. Même si le modèle prédictif est très exact, les chercheurs croient que de nombreux patients pourraient être mal classés. Ils insistent sur l'importance de l'aspect rentabilité d'un modèle prédictif. Un résultat faussement négatif peut exposer les patients à de plus grands risques périopératoires (une baisse d'efficacité), tandis qu'un résultat faussement positif peut les soumettre inutilement à une technique d'intubation de remplacement (une hausse possible de coût).

Des méthodes multifactorielles d'analyse statistique sont largement utilisées et peuvent être utiles pour comprendre des relations complexes. La régression logistique, choisie par Karkouti et coll., est l'une de ces nombreuses méthodes utiles pour des résultats dichotomiques. D'autres comprennent des analyses discriminantes et classificatoires. ${ }^{8}$ Le choix de la méthode appropriée dépend surtout de l'application. Il est important que les prémisses de base et les limites de la méthode choisie soient comprises. Dans le cas de la régression logistique, on doit interpréter les coefficients estimés, être conscient de la possibilité de colinéarité et investiguer la validité de l'ajustement de manière appropriée. Ayant dévoilé leurs limites, ces méthodes peuvent fournir une prédiction fiable. Des études ont montré que l'efficacité et la précision d'une prédiction peuvent être améliorées en utilisant un critère ou une règle explicite. ${ }^{9}$ Une définition claire de l'issue recherchée (déterminer une difficulté d'intubation), une constatation prédictive exacte (le score de Mallampatti) et une évaluation à l'aveugle du résultat (exempte de biais) ont été proposées. Ces règles peuvent contribuer à l'exactitude, mais le risque d'une erreur n'est pas écarté. Selon le seuil choisi, Wilson et coll. ${ }^{10}$ ont montré que les règles peuvent permettre la détection d'un plus grand nombre d'examens laryngoscopiques «difficiles» (forte sensibilité) au prix de la fausse identification d'un plus grand nombre d'examens sans difficulté (faible spécificité). De même, Jacobson et coll. ${ }^{1}{ }^{1}$ ont montré qu'en augmentant la spécificité des tests, le nombre de faux positifs peut être réduit. Malheureusement, cela peut laisser de côté des cas d'examen laryngoscopique et d'intubation difficiles. Idéalement, nous souhaitons que la méthode prédictive nous donne à la fois un indice élevé de sensibilité et de spécificité. Nous devons toutefois faire un compromis et, en conséquence, nous préoccuper du genre d'erreur que nous jugeons la plus importante. Est-ce à dire qu'il est plus acceptable d'identifier correctement des patients dont l'examen laryngoscopique est difficile même si, de cette manière, on inclut faussement quelques patients dont l'examen est facile, plutôt que d'exclure quelques patients difficiles à examiner ? Même si la majorité s'accorde pour dire que le prix d'une mise à l'écart de quelques cas d'intubation difficile est de loin plus élevé que la fausse identification de difficultés, on peut réduire le stress considérable qu'un patient doit subir lors de l'utilisation d'une technique d'intubation de remplacement comme la fibroscopie vigile. De toute évidence, le clinicien doit choisir ce qui est le plus indiqué pour un patient en particulier sans négliger la sécurité.

On doit rappeler qu'il n'y a pas de prédiction parfaite des résultats, mais la prédiction nous aide à planifier des stratégies d'intervention dans des situations problématiques. La fiabilité repose sur l'adhésion à un critère explicite et à un certain réalisme. Tout au plus, il y a une grande variabilité individuelle de mesure des paramètres d'intubation. Les outils de prédiction sont utiles mais seulement lorsqu'on les utilise avec un bon jugement clinique. Nous devons tous reconnaître l'inévitabilité d'une erreur prédictive et nous préparer au pire scénario, comme l'impossibilité d'une ventilation et d'une oxygénation adéquates. Nous devons disposer d'une variété de techniques d'intubation de rechange et être toujours prêts à l'inévitable erreur. Ce fut plus facile pour Noé. On lui a dit ce qu'il fallait faire et comment. Nous n'avons pas cette chance.

\section{References}

1 Mallampati SR, Gatt SP, Gugino LD, et al. A clinical sign to predict difficult tracheal intubation: a prospective study. Can Anaesth Soc J 1985; 32: 429-34.

2 Laplace E, Benefice S, Marti Flich J, Patrigeon RG, Combourieu E. Difficult intubation: a prospective evaluation of the Mallampati and Wilson tests. (French) Cah Anesthesiol 1995; 43: 205-8.

3 Bergler W, Maleck W, Baker-Schreyer A, Ungemach J, Petroianu G, Hormann K The Mallampati Score. Prediction of difficult intubation in otolaryngologic laser surgery by Mallampatti Score. (German) Anaesthesist 1997; 46: 437-40.

4 Naguib M, Malabarey T, AlSatli RA, Al Damegh S, Samarkandi $A H$. Predictive models for difficult laryn- 
goscopy and intubation. A clinical, radiologic and three-dimensional computer imaging study. Can J Anesth 1999; 46: 748-59.

5 Rose DK, Coben MM. The airway: problems and predictions in 18,500 patients. Can J Anaesth 1994; 41: 372-83.

6 El-Ganzouri AR, McCarthy RJ, Tuman KJ, Tanck EN, Ivankovich $A D$. Preoperative airway assessment: predictive value of a multivariate risk index. Anesth Analg 1996; 82: 1197-204.

7 Kartouti K, Rose K, Wigglesworth D, Cohen MM.

Predicting difficult intubation: a multivariable analysis. Can J Anesth 2000; 47: 730-39.

8 Fisher LD, van Belle G Biostatistics. New York: John Wiley \& Sons, Inc., 1993.

9 Wasson JH, Sox HC, Neff RK, Goldman L. Clinical prediction rules. Applications and methodological standards. N Engl J Med 1985; 313: 793-9.

10 Wilson ME, Spiegelhalter D, Robertson JA, Lesser P. Predicting difficult intubation. Br J Anaesth 1988; 61: 211-6.

11 Jacobsen J, Jensen E, Waldau T, Poulsen TD.

Preoperative evaluation of intubation conditions in patients scheduled for elective surgery. Acta Anaesthesiol Scand 1996; 40: 421-4. 